\title{
RELAÇÕES FENOTÍPICAS E GENOTÍPICAS ENTRE COMPONENTES DE PRODUÇÃO EM CANA-DE-AÇÚCAR (1)
}

\author{
FÁBIO MEDEIROS FERREIRA (2,7); WILLIAN SILVA BARROS $\left({ }^{3,7}\right)$, FELIPE LOPES DA SILVA $\left({ }^{2,7}\right)$; \\ MÁRCIO HENRIQUE PEREIRA BARBOSA $\left({ }^{4,8}\right)$; COSME DAMIÃO CRUZ $(5,8)$; \\ IRLANE TOLEDO BASTOS $\left({ }^{6}\right)$
}

\begin{abstract}
RESUMO
Visando quantificar os efeitos diretos e indiretos de componentes de produção sobre as variáveis tonelada de cana por hectare $(\mathrm{TCH})$ e massa média de colmos (MMC) foram realizadas análises de trilha fenotípicas e genotípicas. Dezessete famílias híbridas foram avaliadas no delineamento experimental em blocos casualizados com quatro repetições, no Centro de Pesquisa e Melhoramento da Cana-de-Açúcar (CECA), da Universidade Federal de Viçosa, com o plantio realizado em janeiro de 2000. Foram definidas como componentes de produção as variáveis: altura de colmos (AC), diâmetro de colmos (DC) e número de colmos (NC). As variáveis MMC e NC podem ser utilizadas na seleção indireta de famílias mais produtivas, uma vez que tiveram efeitos diretos positivos e elevados sobre TCH. Apesar de AC e DC estarem altamente correlacionadas com MMC, apenas DC mostrou elevado efeito genético direto sobre MMC, sendo uma variável importante a ser considerada na seleção indireta de famílias com maior massa média de colmos.
\end{abstract}

Palavras-chave: correlações genotípicas, correlações fenotípicas, análise de trilha.

\section{ABSTRACT \\ PHENOTYPIC AND GENETIC RELATIONSHIPS AMONG YIELD COMPONENTS IN SUGARCANE}

Phenotypic and genetic path analyses were carried out in order to quantify direct and indirect effects of production components on the variable ton of stalks per hectare (TSH) and mean stalk weight (MSW). Seventeen hybrid families were evaluated on a randomized complete block design with four replicates, at the Centro de Pesquisa e Melhoramento da Cana-de-Açúcar - CECA of the Universidade Federal de Viçosa, Minas Gerais State. Planting was carried out in January 2000. The following variables were defined as production components: stalk height (SH), stalk diameter (SD) and stalk number (SN). The results indicated that the variables MSW and SN can be used for indirect selection of high yielding families, since they showed positive and high direct effects on TSH. Despite SH and SD showed high correlation with MSW, only SD showed high direct genetic effect on MSW, demonstrating the importance of this variable for indirect selection of families with higher mean stalk weight.

Key words: genetic correlations, correlations, path analysis.

( $\left.{ }^{1}\right)$ Recebido para publicação em 12 de dezembro de 2006 e aceito em 11 de junho de 2007.

$\left({ }^{2}\right)$ Doutorando em Genética e Melhoramento, Universidade Federal de Viçosa, 36570-000, Viçosa (MG). E-mail: fmferreira@yahoo.com.br; lipells@yahoo.com.br

(3) Pós-Doutorando em Genética e Melhoramento, Universidade Federal de Viçosa. E-mail: wsbarros@hotmail.com

$\left({ }^{4}\right)$ Departamento de Fitotecnia, Universidade Federal de Viçosa. E-mail: barbosa@ufv.br

$\left({ }^{5}\right)$ Departamento de Biologia Geral, Universidade Federal de Viçosa. E-mail: cdcruz@ufv.br (*) Autor correspondente.

$\left({ }^{6}\right)$ Doutora em Genética e Melhoramento pela Universidade Federal de Viçosa. E-mail: ibastos@ufv.br

$\left({ }^{7}\right)$ Bolsista do CNPq.

$\left({ }^{8}\right)$ Com bolsa de produtividade científica do CNPq. 


\section{INTRODUÇÃO}

Entender as relações existentes entre variáveis é de suma importância, visto que a obtenção de ganhos genéticos e a definição dos melhores genótipos são, muitas vezes, dirigidas a um conjunto de variáveis agronômicas e comerciais. Além disso, o conhecimento dessas relações permite que em uma variável principal, caracterizada por baixa herdabilidade e/ou dificuldades de medição, seja praticada a seleção com base em outra(s), possibilitando ao melhorista obter progressos mais rápidos em relação ao uso da seleção direta.

O grau de associação linear entre duas variáveis quaisquer pode ser definido pela correlação de Pearson. Nos estudos genéticos, a correlação tem basicamente duas origens: genética e ambiental. Segundo FALCONER (1987), a correlação genética é ocasionada principalmente pelo pleiotropismo (propriedade pela qual um gene condiciona mais de um caráter simultaneamente), além do desequilíbrio de ligação (associação não aleatória entre alelos de diferentes locos). Entretanto a correlação não é uma medida de causa e efeito. A interpretação direta das suas magnitudes pode resultar em equívocos na estratégia de seleção, pois uma alta correlação entre duas variáveis pode ser resultado do(s) efeito(s) de outra(s) sobre elas. Sugere-se estudo de correlações parciais, correlações canônicas e análise de trilha, para o melhor entendimento dos fenômenos de associação entre variáveis.

A análise de trilha (path analysis), desenvolvida por Wright $(1921,1923)$ e pormenorizada por Li (1975), permite desdobrar os coeficientes de correlação em efeitos diretos e indiretos sobre uma variável principal, cujas estimativas são obtidas por meio de equações de regressão considerando a padronização das variáveis. Além do entendimento das relações de causa e efeito entre as variáveis, o desdobramento das correlações é dependente do conjunto de variáveis estudadas, normalmente estabelecido pelo conhecimento prévio do melhorista de sua importância e de possíveis inter-relações definidas em diagramas de trilha (CRUz et al., 2003).

A produção de colmos e açúcar por hectare são as variáveis de maior relevância nos programas de melhoramento genético de cana-de-açúcar. Trata-se de variáveis de herança complexa e que provavelmente apresentam efeitos pleiotrópicos com outras variáveis. A literatura apresenta alguns estudos sobre as relações entre variáveis importantes para a cultura da cana, via análise de trilha (Miller, 1977; KANG et al., 1983; Gravois et al., 1990; Gravois e Milligan, 1992; SUKHCHAIN et al., 1997). Entretanto, estudos desta natureza ainda são necessários, visto que diferentes estruturas genéticas populacionais têm sido consideradas.

Neste contexto, o objetivo principal foi quantificar os efeitos diretos e indiretos de componentes de produção sobre a variável tonelada de colmos por hectare e massa média de colmos, bem como identificar possíveis critérios de seleção indireta, por intermédio da avaliação de famílias híbridas.

\section{MATERIAL E MÉTODOS}

Foram avaliadas 17 famílias resultantes dos cruzamentos dialélicos entre os genótipos SP80-4439, SP84-5124, SP84-1192 e SP84-2025 com os genótipos SP80-3280, SP82-3530, SP83-1483, SP85-7227 e SP88721 , realizados em condições de campo, no município de Camamu, Bahia. Para prevenir a autofecundação, todas as inflorescências fêmeas foram emasculadas usando tratamento com água quente (MACHADO JÚNIOR et al., 1995).

As progênies foram enviadas ao Centro de Pesquisa e Melhoramento da Cana-de-Açúcar (CECA), da Universidade Federal de Viçosa, cuja área experimental se localiza no município de Oratórios, Minas Gerais, com latitude $20^{\circ} 25^{\prime} S$; longitude $42^{\circ} 48^{\prime} \mathrm{W}$; altitude $494 \mathrm{~m}$; solo LVE. A obtenção e o transplantio de seedlings foram realizados conforme descrito por BARBOSA e Silveira (2000). O plantio foi feito em janeiro de 2000 e em julho de 2000 quando todas as plantas foram cortadas manualmente com facão, e os seedlings submetidos à seleção natural para capacidade de rebrota em condições ambientais desfavoráveis, isto é, época seca e fria. Utilizou-se como bordadura, nas laterais do experimento, a variedade RB72454. A adubação empregada foi de 225 g por sulco do formulado contendo $5 \%$ de N, $25 \% \mathrm{P}_{2} \mathrm{O}_{5}$ e $25 \%$ de $\mathrm{K}_{2} 0$.

O experimento foi delineado em blocos casualizados com quatro repetições, sendo cada parcela composta por dois sulcos espaçados de 1,40 m. Cada sulco foi constituído por dez plantas eqüidistantes a 0,5 m. Em abril de 2001 coletaram-se os dados em cana-soca.

As variáveis avaliadas foram: a) altura média de colmos (AC) em metros, mensurando-se um colmo de cada touceira, desde a base do colmo até o primeiro dewlap visível; b) diâmetro de colmos (DC) em metros, com a amostragem feita no quinto entrenó, contado da base do colmo para o ápice, mensurando-se um colmo de cada touceira com paquímetro; c) número de colmos por parcela (NC); 4) massa média de colmos por parcela (MMC) em quilogramas, amostrada 
aleatoriamente por 20 colmos por sulco; 5) toneladas de colmos por hectare $(\mathrm{TCH})$, com pesagem de toda a parcela utilizando-se dinamômetro.

Estimativas de herdabilidade no sentido amplo, coeficientes de variação ambiental e correlações genotípicas e fenotípicas das variáveis sob estudo foram obtidos conforme apresentado por CRUZ et al. (2003).

O estimador de MMC, descrito por CHANG e Milligan (1992), é definido por:

$$
\mathrm{MMC}=d x \pi \times A C \times\left(\frac{D C}{2}\right)^{2}
$$

em que $d$ é a densidade específica do colmo. Chang e Milligan (1992) sugeriram utilizar para p o valor de $1.000 \mathrm{~kg} \mathrm{~m}^{-3}$, que é equivalente a $1,0 \mathrm{~g} \mathrm{~cm}^{-3}$.

Utilizando este estimador de MMC é possível predizer o valor de $\mathrm{TCH}$ por meio da expressão:

$$
\mathrm{TCH}=d x \pi \times N C \times A C \times\left(\frac{D C}{2}\right)^{2} x \frac{1}{100 \times t p}
$$

em que tp é o tamanho da parcela $\mathrm{em}^{2}$.

Observa-se que a relação das variáveis explicativas NC, AC e DC e as variáveis TCH e MMC é estruturalmente multiplicativa. Por conseguinte, foi estabelecida a transformação logarítmica dos dados originais, de modo a obter o seguinte modelo aditivo de regressão linear múltipla:

$$
\begin{aligned}
& \log \mathrm{TCH}=\mathrm{p}_{\mathrm{ac}} \log (\mathrm{AC})+\mathrm{p}_{\mathrm{dc}} \log (\mathrm{DC})+\mathrm{p}_{\mathrm{nc}} \log (\mathrm{NC})+p_{\varepsilon} u \\
& \log \mathrm{PMC}=\mathrm{p}_{\mathrm{ac}} \log (\mathrm{AC})+\mathrm{p}_{\mathrm{dc}} \log (\mathrm{DC})+p_{\varepsilon} u
\end{aligned}
$$

em que $p_{i}$ é a medida do efeito direto (ou coeficiente de trilha) da i-ésima variável explicativas (ou componentes de produção: AC, DC e NC) sobre a variável principal (TCH e MMC); é o efeito direto de outras variáveis, não consideradas no modelo, sobre a variável principal; é o erro padronizado associado ao modelo; AC, DC, NC, MMC e TCH são os valores observados padronizados.

O diagrama causal do inter-relacionamento das variáveis analisadas é ilustrado pela Figura 1.

Os coeficientes de trilha foram estimados a partir do sistema de equações normais, $X^{\prime} X \beta=X^{\prime} Y$, em que $X^{\prime} X$ é a matriz de correlações genotípicas ou fenotípicas entre as variáveis explicativas do modelo matemático; é o vetor dos estimadores dos coeficientes de trilha; $\beta$ e $X^{\prime} Y$ é a matriz de correlações genotípicas ou fenotípicas entre a variável principal e cada variável explicativa do modelo.

Decompondo-se as correlações de TCH e MMC com os componentes de produção, tem-se:

$$
r_{i, T C H}=p_{i}+\sum_{j \neq i}^{n} p_{i} r_{i i^{\prime}} \text { e } r_{i, P M C}=p_{i}+\sum_{j \neq i}^{n} p_{i} r_{i i^{\prime}}
$$

em que, $\mathrm{r}_{\mathrm{i}, \mathrm{TCH}}(\mathrm{ou})$ é a correlação entre a variável TCH (ou MMC) com a i-ésima variável explicativa (AC, DC, NC); $\mathrm{p}_{\mathrm{i}} \mathrm{r}_{\mathrm{ii}}$ é a medida do efeito indireto da variável $i$, via variável $i^{\prime}$ (para todo $\left.i \neq i^{\prime}\right)$, sobre TCH (ou MMC).
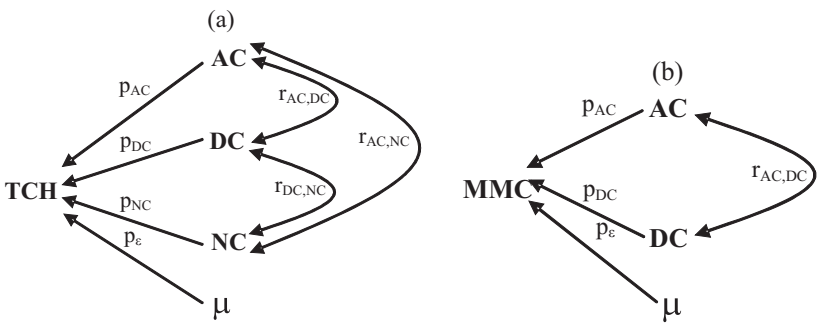

Figura 1. Diagrama causal ilustrativo dos efeitos diretos e indiretos dos componentes de produção: (a) altura de colmos (AC), diâmetro de colmos (DC), número de colmos (NC) sobre toneladas de colmos por hectare $(\mathrm{TCH})$; (b) altura de colmos (AC), diâmetro de colmos (DC) sobre massa média de colmos (MMC). A seta unidirecional indica efeito direto de cada variável explicativa sobre a principal; a seta bidirecional representa a interdependência de duas variáveis explicativas, cuja magnitude é quantificada pela correlação genotípica ou fenotípica (r.) entre elas.

Antes da execução da análise de trilha realizou-se o diagnóstico de multicolinearidade nas matrizes de correlações genotípicas e fenotípicas, de acordo com os critérios indicados por MonTGOMERY e Peck (1981), resultando em colinearidade fraca. Todas as análises foram executadas pelo programa GENES (CRUZ, 2006).

\section{RESULTADOS E DISCUSSÃO}

Segundo o critério estabelecido por Gomes (1990), os coeficientes de variação (CV) para tonelada de colmos por hectare (TCH) e número de colmos (NC) foram elevados (25,47 e $27,06 \%$ respectivamente). Por outro lado, obtiveram-se baixas estimativas de $\mathrm{CV}$ para altura de colmos (AC) $(5,25 \%)$ e diâmetro de colmos (DC) $(5,38 \%)$. Para massa média do colmo (MMC) a estimativa de CV foi igual a $11,24 \%$. Em geral, a precisão experimental pôde ser considerada adequada, graças à significância $(P<0,05)$ da variabilidade genética entre as famílias testadas, dados não apresentados. 
Em culturas de propagação assexuada como a cana-de-açúcar, a informação de herdabilidade no sentido amplo é importante desde que toda a variabilidade genética seja utilizada entre as gerações de seleção assexuada (HogArTH, 1971). Nas variáveis $\mathrm{NC}$ e TCH, observaram-se as menores estimativas de herdabilidade no sentido amplo $\left(\mathrm{h}^{2}=47,14\right.$ e $47,33 \%$ respectivamente). Já para as variáveis $\mathrm{AC}, \mathrm{DC}$ e MMC as estimativas de herdabilidade foram de $72,44,62,01$ e $80,04 \%$, respectivamente, considerando-as de magnitude satisfatórias (dados não apresentados). As estimativas de herdabilidade têm variado de 0,21 a 0,84 para $\mathrm{AC} ; 0,30$ a 0,94 para $\mathrm{DC} ; 0,13$ a 0,82 para NC; 0,48 a 0,81 para MMC, conforme as populações estudadas e seus contextos genéticos (CESNIK e VenCOVSKY, 1974; Hogarth, 1977; Hogarth et al., 1981; KANG et al., 1983; SKINNER et al., 1987).

No geral, as estimativas das correlações fenotípicas e genotípicas foram concordantes quanto ao sinal e os valores foram bem próximos, com superioridade atribuída às correlações genotípicas (Tabela 1). Utilizou-se o artifício de assumir como 0,9999 o coeficiente de correlação entre MMC e AC, cuja estimativa foi superior à unidade. Nos casos em que há uma proximidade na magnitude das correlações fenotípicas e genotípicas, reflete-se a pequena influência ambiental sobre a associação de duas variáveis. As correlações genotípicas entre TCH x NC $(0,49)$ e TCH x AC $(0,55)$ não foram significativas $(\mathrm{P}>0,05)$. A maioria das correlações fenotípicas e genotípicas entre os componentes de produção (AC, DC, NC) com TCH e MMC foram altas ou moderadas e positivas, a exceção de NC com MMC, à semelhança dos resultados obtidos por KANG et al. (1983) e REDDY e REDDI (1986).

KANG et al. (1983) argumentam que, do ponto de vista prático, é mais útil inferir sobre os efeitos diretos genotípicos na tomada de decisão acerca do critério de seleção mais adequado. Contudo, SHuKLA et al. (1998) alegam que variáveis correlacionadas genotipicamente, porém não fenotipicamente, podem não ser de valor prático na seleção, pois esta é geralmente baseada no fenótipo.

$\mathrm{Na}$ tabela 1 observa-se que a variável NC apresenta estimativas de correlação genotípica e fenotípica moderadas com TCH, assim como seus efeitos diretos (Tabela 2). O efeito direto fenotípico de $\mathrm{NC}(0,69)$ foi superior ao efeito direto genotípico $(0,49)$ sobre $\mathrm{TCH}$.

A variável AC apresentou efeitos diretos pequenos sobre $\mathrm{TCH}$, sendo o efeito direto genotípico negativo $(-0,39)$. O efeito indireto genotípico de AC via DC foi alto, embora a correlação genotípica entre TCH e AC tenha sido não significativa. Os efeitos diretos (genotípico e fenotípico) de DC sobre TCH foram altos e positivos. SIDWELL et al. (1976) argumentam que os efeitos diretos fenotípicos e genotípicos são de magnitudes semelhantes, porém estariam sendo pouco afetados pelo efeito genético não-aditivo ou ambiental, ao contrário do que se observou neste estudo (Tabela 2), em que se destacam o diâmetro de colmos seguido do número de colmos como as variáveis que mais contribuíram para explicar TCH. Nos trabalhos de JAMes (1971), Miller e James (1974) e KANG et al. (1983) verificou-se uma contribuição equivalente do número de colmos e diâmetro de colmos sobre TCH, seguidos pela altura de plantas. SuKHCHAIN et al. (1997) também obtiveram altos efeitos diretos de $\mathrm{NC}$ sobre $\mathrm{TCH}$, sugerindo a seleção de clones para elevação da produção de colmos com base nesta variável. Em virtude da baixa e negativa associação entre NC e DC, sugere-se ao melhorista a aplicação de técnicas de seleção simultânea para essas duas variáveis quando se deseja melhorá-las concomitantemente.

Tabela 1. Estimativas dos coeficientes de correlação fenotípica e genotípica (entre parênteses) entre as variáveis altura de colmos (AC), diâmetro de colmos (DC), número de colmos (NC), massa média de colmos (MMC) e toneladas de cana por hectare $(\mathrm{TCH})$, avaliadas em famílias de cana-de-açúcar

\begin{tabular}{lcccc}
\hline Variáveis & MMC & AC & DC & TCH \\
\hline NC & $-0,1636$ & $-0,1714$ & $-0,0977$ & $0,6029^{* *}$ \\
& $(-0,1773)$ & $(-0,3481)$ & $(-0,1065)$ & 0,4893 \\
MMC & & $0,8913^{* *}$ & $0,7119^{* *}$ & $0,5214^{*}$ \\
& & $\left(0,9999^{* *(1)}\right.$ & $\left(0,9087^{* *}\right)$ & $\left(0,9498^{* *}\right)$ \\
AC & & $0,7129^{* *}$ & $0,5111^{*}$ \\
& & & $\left(0,8540^{* *}\right)$ & 0,5505 \\
DC & & & $0,6458^{* *}$ \\
& & & & $\left(0,9177^{* *}\right)$ \\
\hline
\end{tabular}

*,** Significativo ao nível de 0,05 e 0,01 pelo teste de Mantel com 1.000 simulações respectivamente.

$\left({ }^{1}\right)$ A correlação genotípica entre MMC e AC foi superior à unidade, sendo atribuída a esta correlação valor igual a 0,9999.

Os coeficientes de trilha fenotípicos (Tabela 2) explicaram bem as variações em $\mathrm{TCH}$, como indica o alto valor de determinação do modelo $\left(R^{2}=0,89\right)$ e pelo efeito residual pequeno $(0,32)$, o que reflete a excelente contribuição das variáveis do modelo para a produção de colmos. Melhor ainda foi o modelo genotípico, que explicou em $100 \%$ a variação em $\mathrm{TCH}$.

BRESSIANi et al. (2001) relata que os componentes envolvidos na tonelagem de açúcar são a TCH e o teor de açúcar do colmo (Brix). Ambos são de grande importância, sendo possível pode ser 
ainda subdividir a TCH nos componentes, número de colmos por hectare e massa de colmos, este último sendo composto pelo diâmetro, altura e densidade do colmo.

Tabela 2. Análise de trilha fenotípica e genotípica dos componentes de produção número de colmos (NC), altura de colmos (AC) e diâmetro de colmos (DC) sobre toneladas de cana por hectare $(\mathrm{TCH})$

\begin{tabular}{lcc}
\hline Variáveis & Fenotípica & Genotípica \\
\hline NC & & \\
Efeito direto sobre TCH & 0,6978 & 0,4911 \\
Efeito indireto via AC & $-0,0424$ & 0,1375 \\
Efeito indireto via DC & $-0,0525$ & $-0,1392$ \\
Total & 0,6029 & 0,4893 \\
AC & & \\
Efeito direto sobre TCH & 0,2475 & $-0,3949$ \\
Efeito indireto via NC & $-0,1196$ & $-0,1709$ \\
Efeito indireto via DC & 0,3832 & 1,1164 \\
Total & 0,5111 & 0,5505 \\
DC & & \\
Efeito direto sobre TCH & 0,5375 & 1,3072 \\
Efeito indireto via NC & $-0,0682$ & $-0,0523$ \\
Efeito indireto via AC & 0,1764 & $-0,3373$ \\
Total & 0,6458 & 0,9177 \\
Coeficiente de determinação $\left(\mathrm{R}^{2}\right)$ & 0,8944 & 1,2225 \\
Efeito da variável residual & 0,325 & 0 \\
\hline
\end{tabular}

Neste contexto, uma segunda análise de trilha foi realizada, definindo-se um modelo de regressão em que MMC é dado como a variável principal e AC e DC as variáveis explicativas (Figura 1b). A variável MMC é altamente correlacionada com AC e DC (Tabela 1). No entanto, os efeitos diretos de DC sobre MMC foram pequenos (Tabela 3). Em contrapartida, a variável AC apresentou valores elevados dos coeficientes de trilha $(0,78$ e 0,83$)$. Ressaltam-se os altos valores dos efeitos indiretos (fenotípico e genotípico) de DC via AC, o que corrobora a maior importância de AC neste modelo. Ao contrário, Gravois e Milligan (1992) obtiveram efeito direto genotípico de DC de maior magnitude quando comparado a AC. Os autores consideraram as variáveis DC e AC independentes, em virtude dos baixos efeitos indiretos apresentados. No diagrama causal por eles proposto, incluíram as variáveis Brix e fibra, porém ambas com baixos efeitos diretos.

Os coeficientes de determinação $\left(R^{2}\right)$ iguais a 0,81 e 1,01 e o baixo e nulo efeito residual representam satisfatoriamente a contribuição de DC e AC para a massa de colmos (Tabela 3).
Tabela 3. Análise de trilha fenotípica e genotípica dos componentes de produção altura de colmos (AC) e diâmetro de colmos (DC) sobre o massa média de colmos (MMC)

\begin{tabular}{lcc}
\hline Variáveis & Fenotípica & Genotípica \\
\hline AC & & \\
Efeito direto sobre MMC & 0,7804 & 0,8271 \\
Efeito indireto via DC & 0,1109 & 0,1728 \\
Total & 0,8913 & 0,9999 \\
DC & & \\
Efeito direto sobre MMC & 0,1555 & 0,2024 \\
Efeito indireto via AC & 0,5564 & 0,7063 \\
Total & 0,7119 & 0,9087 \\
Coeficiente de determinação $\left(\mathrm{R}^{2}\right)$ & 0,8063 & 1,0109 \\
Efeito da variável residual & 0,4401 & 0 \\
\hline
\end{tabular}

Estudos desta natureza são relevantes, pois nas relações de causa e efeito entre os componentes de produção em cana-de-açúcar têm havido algumas variações na literatura, sob os diferentes contextos genéticos empregados.

\section{CONCLUSÕES}

1. A seleção das melhores famílias quanto à produção de cana-de-açúcar pode ser realizada com base no diâmetro de colmos e número de colmos.

2. A variável altura de colmos foi a que melhor contribuiu para a massa média de colmos.

\section{AGRADECIMENTOS}

Ao $\mathrm{CNPq}$ e à FAPEMIG pelo suporte financeiro e pela concessão de bolsa de estudo. À COPERSUCAR pelo fornecimento das sementes e realização dos cruzamentos no município de Camamu, Bahia.

\section{REFERÊNCIAS}

BARBOSA, M.H.P.; SILVEIRA, L.C.I. Metodologias de seleção, progressos e mudanças no programa de melhoramento genético da cana-de-açúcar da Universidade Federal de Viçosa. STAB, Açúcar, Álcool e Subprodutos, Piracicaba, v.18, n.3, p.30-32, 2000.

BRESSIANI, J.A.; BURNQUIST, W.L.; FUZATTO, S.R.; BONATO, A.L.V.; GERALDI, I.O. Combining ability in eight selected clones of sugarcane (Saccharum sp.). Crop Breeding and Applied Biotechnology, Londrina, v.2, n.3, p.411-416, 2001. 
CESNIK, R.; VENCOVSKY, R. Expected response to selection, heritability, genetic correlations and response to selection of some characters in sugarcane. In: PROCEEDINGS OF INTERNATIONAL SOCIETY OF SUGAR CANE TECHNOLOGISTS CONGRESS, 1974, Durban. Proceedings... Durban, South Africa: Hayne and Gibbon Limited, 1974. p.96-101.

CHANG, Y.S.; MILLIGAN S.B. Estimating the potential of sugarcane families to procude elite genotypes using univariate cross prediction methods. Theoretical and Applied Genetics, Berlin, v. 84, p. 662-671, 1992.

CRUZ, C.D. Programa Genes: estatística experimental e matrizes. 1.ed. Viçosa: Editora UFV, 2006. 285p.

CRUZ, C.D.; REGAZZI, A.J.; CARNEIRO, P.C.S. Modelos biométricos aplicados ao melhoramento genético. v.2. Viçosa: Editora UFV, 2003, 585p.

FALCONER, D.S. Introdução à genética quantitativa. Viçosa, Imprensa Universitária da UFV, 1987. 279p.

GOMES, F.P. Curso de estatística experimental. 13.ed. Piracicaba: USP, 1990. 467p.

GRAVOIS, K.A; MILLIGAN, S.B. Genetic relationships between fiber and sugarcane yield components. Crop Science, Madison, v.32, n.1, p.62-67, 1992.

GRAVOIS, K.A.; MILLIGAN, S.B.; MARTIN, F.A. Role of pith, tube, and stalk density in determining sugarcane sucrose content and stalk weight. Theoretical and Applied Genetics, Berlin, v.79, n.2, p.273-277, 1990.

HOGARTH, D.M. Quantitative inheritance studies in sugarcane. I Estimation of variance components. Australian Journal Research, Victoria, v.22, n.1, p.93-102, 1971.

HOGARTH, D.M. Quantitative inheritance studies in sugarcane. III The effect of competition and violation of assumptions on estimation of genetic variance components. Australian Journal Research, Victoria, v.28, n.2, p.257-268, 1977.

HOGARTH, D.M.; WU, K.K.; HEINZ, D.J. Estimating genetic variance in sugarcane using a factorial cross design. Crop Science, Madison, v.21, n.1, p.21-25, 1981.

JAMES, N.I. Yield components in random and selected sugarcane populations. Crop Science, Madison,v.11, n.6, p.906$908,1971$.

KANG, M.S.; MILLER, J.D.; TAI, P.Y.P. Genetic and phenotypic path analysis and heritability in sugarcane. Crop Science, Madison, v.23, n.4, p.643-647, 1983.

LI, C. C. Path analysis: aprimer. Pacific Grove: Boxwood, 1975. $346 \mathrm{p}$.

MACHADO JUNIOR, G.R.; WALKER, D.I.; BRESSIANI, J.A.; DA SILVA, J.A.G. Emasculation of sugarcane tassels using hot water. In: INTERNATIONAL SOCIETY OF SUGAR CANE TECHNOLOGISTS CONGRESS, 22., 1995 Cartagena. Proceedings... Cali: Tecnicaña, 1995. p. 346-352.
MILLER, J.D.; JAMES, N.I. The influence of stalk densityon cane yield. In: INTERNATIONAL SOCIETY OF SUGARCANE TECHOLOGY CONGRESS, 1974. South Africa. Proceedings... Durban, South Africa : Hayne and Gibbon Limited, 1974. p. 177-184.

MILLER, J.D. Combining ability and yield component analyses in a five-parent diallel cross in sugarcane. Crop Science, Madison, v.17, n.4, p.545-547, 1977.

MONTGOMERY, D.C.; PECK, E.A. Introduction to linear regression analysis. New York: John Wiley \& Sons, 1981. 504p.

REDDY, C.R.; REDDI, M.V. Degree of genetic determination, correlation and genotypic and phenotypic path analysis of cane and sugar yield in sugarcane. Indian Journal of Genetics and Plant Breeding, New Delhi, v.46, n.3, p.550-557, 1986.

SHUKLA, S.; SINGH, K.; PUSHPENDRA. Correlation and path coefficient analysis of yield and its components in soybean (Glycine max L. Merrill.). Soybean Genetics Newsletter, Ames, v.25, n.1, p.67-70, 1998.

SIDWELL, R.J.; SMITH, E.L.; MCNEW, R.W. Inheritance and interrelationships of grain yield and selected yield-related traits in hard red winter wheat cross. Crop Science, Madison, v.16, n.5, p.650-654, 1976.

SKINNER, J.C.; HOGARTH, D.M.; WU, K.K. Selection methods, criteria and indices. In: Heinz, D.J. (Ed.). Sugarcane improvement through breeding. Elsevier, Amsterdam, 1987. p.409-453.

SUKHCHAIN; SANDHU, D.; SAINI, G.S. Inter-relationships among cane yield and commercial cane sugar and their component traits in autumn plant crop of sugarcane. Euphytica, Dordrecht, v.95, n.1, p.109-114, 1997.

WRIGHT, S. Correlation and causation. Journal of Agricultural Research, Washington, v.20, n.7, p.557-585, 1921.

WRIGHT, S. The theory of path coefficients: a replay to Niles' criticism. Genetics, Austin, v.8, n.3, p. 239-255, 1923. 